\title{
The Munich Compact Light Source: Biomedical Research At a Laboratory-Scale Inverse-Compton Synchrotron X-ray Source
}

Benedikt Günther ${ }^{1,2,3}$, Martin Dierolf ${ }^{1,2}$, Regine Gradl ${ }^{1,2,4}$, Elena Eggl ${ }^{1,2}$, Christoph Jud ${ }^{1,2}$, Lorenz Hehn $^{1,5}$, Stephanie Kulpe ${ }^{1,2}$, Bernhard Gleich ${ }^{1,2}$, Madleen Busse ${ }^{1,2}$, Kaye S. Morgan ${ }^{1,2,4,6}$, Klaus Achterhold $^{1,2}$ and Franz Pfeiffer ${ }^{1,2,5}$

1. Chair of Biomedical Physics, Department of Physics, Technical University of Munich, Garching, Germany

2. Munich School of BioEngineering, Technical University of Munich, Garching, Germany

3. Max-Planck-Institute of Quantum Optics, Garching, Germany

4. Institute for Advanced Studies, Technical University of Munich, Garching, Germany

5. Department of Diagnostic and Interventional Radiology, Technical University of Munich, Munich, Germany

6. School of Physics and Astronomy, Monash University, Melbourne, Australia

Advanced X-ray imaging techniques have been developed in recent years, that provide resolution down to the nanometer-scale, as well as high sensitivity, primarily driven by the brilliant X-ray radiation generated by third generation synchrotron sources. X-rays produced by compact inverse-Compton synchrotron sources (ICS), such as the Munich Compact Light Source (MuCLS), exhibit brilliance in the gap between X-ray tubes and classical synchrotrons [1]. Simultaneously providing a much smaller footprint and being cost-effective compared to the latter, ICS overcome the main limitations for widespread application of advanced imaging techniques in industry and health care. This makes the MuCLS well-suited for various applications in biomedical imaging [2-6], on which we will focus here after a brief review of recent source development at the MuCLS.

We present recent performance upgrades of the MuCLS addressing both, source stability and X-ray flux, followed by a discussion of the resulting improved source parameters. As the MuCLS is based on inverse Compton scattering, a high power laser system is a key component. A new laser amplifier with twice the initial laser power was integrated, more than doubling the initial X-ray flux to $>3 \cdot 10^{10} \mathrm{ph} / \mathrm{s}$ at the highest energy of $35 \mathrm{keV}$ (for initial beam parameters see [1]). In consequence of the increased thermal load by the new amplifier, a system for active thermal stabilization was developed.

X-rays emerging from the MuCLS are guided to two end-stations equipped with complementary set-ups for imaging. A schematic drawing of the beam line is shown in Figure 1. The X-ray beam divergence of $2 \mathrm{mrad}$ creates a higher flux density close to the source. Therefore, the first end-station, where the sample is typically located at a distance of about $4 \mathrm{~m}$ to the X-ray source, is dedicated to micro-tomography and (dynamic) propagation-based phase-contrast imaging. The second end-station, located at a distance of $14.5 \mathrm{~m}$ to the X-ray source, is suited to large samples and optionally contains a Talbot interferometer.

These set-ups can be adapted to different time scales, sample sizes and spatial resolution. In combination with the high brilliance and partial coherence of the MuCLS, this enables the study of many topics of biomedical interest. We present our research in the fields of angiography [4] and (dynamic) K-edge subtraction imaging, detection of micro-fractures [5], phase-contrast imaging applied to mammography, as well as in vivo small-animal respiratory imaging [6]. We are able to demonstrate superior image quality and diagnostic value compared to state of the art clinical procedures and to transfer techniques 
that have so far been limited to large-scale synchrotron facilities, like dynamic small-animal respiratory imaging, shown in Figure 2, into a laboratory.

The techniques introduced above are well-suited to - but not limited to - biomedical applications. For example, the same methodology to detect micro-fractures in bones can be employed for the analysis of carbon fibre composites. In conclusion, a compact inverse-Compton synchrotron source accesses advanced imaging approaches in a laboratory environment providing viable information for medical diagnostics and research as well as non-destructive testing [7].

\section{References:}

[1] E Eggl et al, Journal of Synchrotron Radiation 23 (2016), p. 1137.

[2] K Achterhold et al, Scientific Reports 3 (2013), p. 1313.

[3] E Eggl et al, PNAS 112 (18) (2015), p. 5567.

[4] E Eggl et al, Scientific Reports 7 (2017), p. 42211.

[5] C Jud et al, Scientific Reports 7 (2017), p. 14477.

[6] R Gradl et al, Scientific Reports 7 (2017), p. 4908.

[7] The MuCLS was developed and constructed by Lyncean Technologies Inc. We acknowledge financial support through the Center of Advanced Laser Applications (CALA) and the Cluster of Excellence of the German Research Foundation (DFG) Munich-Centre for Advanced Photonics (MAP).

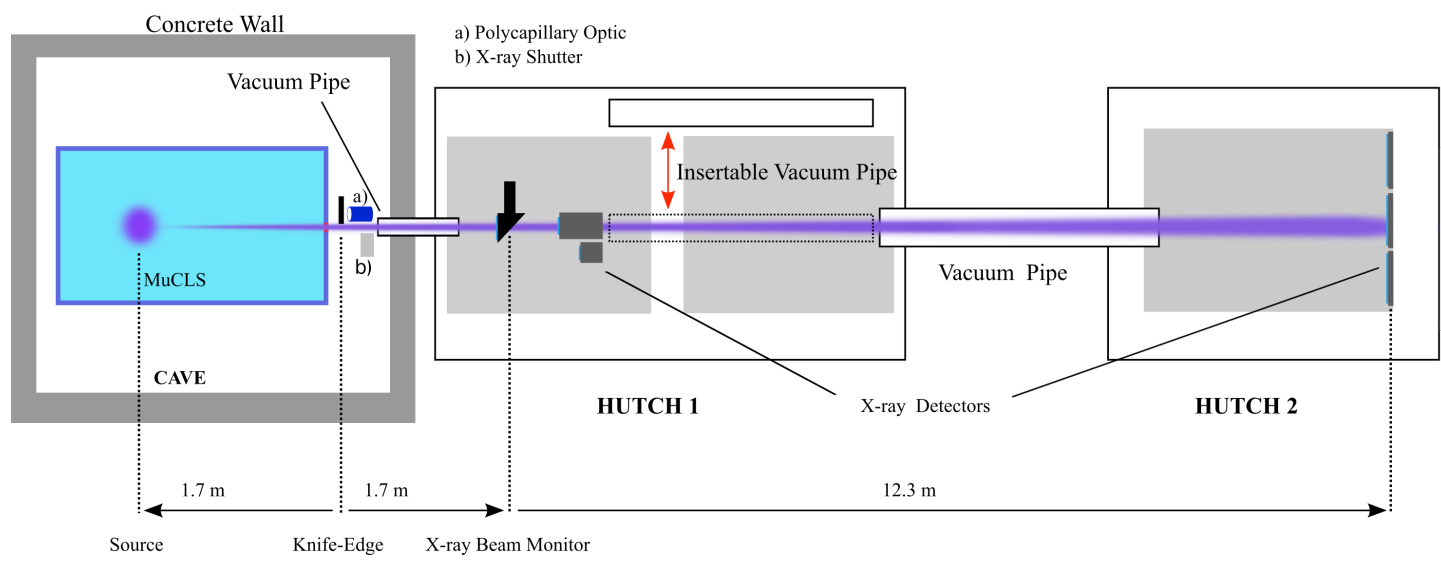

Figure 1. Schematic of the beam line at the MuCLS. The shortest source to sample distance is $3.5 \mathrm{~m}$ and the maximum one is $15.5 \mathrm{~m}$. Figure adapted from D. Cont.
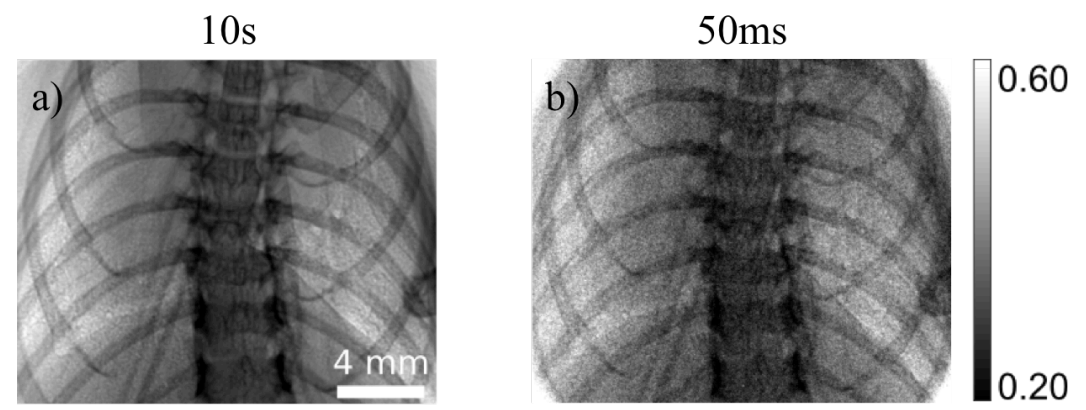

Figure 2. Images of a mouse lung exhibit sufficient image quality at short exposure times (b) compared to long ones (a) enabling dynamic propagation based imaging. Figure adapted from [6]. 